\title{
Efetividade de três esquemas com sulfato ferroso para tratamento de anemia em gestantes
}

\author{
Ariani Impieri de Souza, ${ }^{1}$ Malaquias Batista Filho, ${ }^{1,2}$ \\ Luiz Oscar Cardoso Ferreira ${ }^{3}$ e José Natal Figueirôa ${ }^{1}$
}

Como citar Souza AI de, Batista Filho M, Ferreira LOC, Figueirôa JN. Efetividade de três esquemas com sulfato ferroso para tratamento de anemia em gestantes. Rev Panam Salud Publica. 2004;15(5):313-19.

RESUMO Objetivo. Avaliar a efetividade de três esquemas de tratamento utilizando sulfato ferroso em gestantes anêmicas.

Métodos. O estudo foi desenvolvido no Centro de Atenção à Mulher do Instituto Materno Infantil de Pernambuco, em Recife, no período de maio de 2000 a dezembro de 2001. Realizouse um ensaio clínico, aleatório e cego do ponto de vista laboratorial, utilizando comprimidos de $300 \mathrm{mg}$ de sulfato ferroso para administração de $60 \mathrm{mg}$ de ferro elementar. As gestantes foram alocadas em três grupos de tratamento, conforme a freqüência de administração do ferro: uma vez por semana $(\mathrm{n}=48)$; duas vezes por semana $(\mathrm{n}=53)$; e uma vez ao dia $(\mathrm{n}=49)$. Foram comparadas as concentrações de hemoglobina, volume corpuscular médio e ferritina.

Resultados. Antes da intervenção, os grupos eram homogêneos e apresentaram as seguintes médias e desvios-padrão nas concentrações de hemoglobina: 10,2 $\pm 0,5 \mathrm{~g} / \mathrm{dL}$ para o grupo que recebeu sulfato ferroso uma vez por semana; $10,2 \pm 0,6 \mathrm{~g} / \mathrm{dL}$ para o grupo que recebeu sulfato ferroso duas vezes por semana; e 10,1 $\pm 0,6 \mathrm{~g} / \mathrm{dL}$ para o grupo que recebeu sulfato ferroso uma vez ao dia. As médias de volume corpuscular médio foram, respectivamente: $88,5 \pm 5,0$; $87,6 \pm 5,9 ;$ e $88,7 \pm 5,1 \mathrm{fL}$. As medianas de ferritina foram 30,2;37,1; e 52,9 $\mathrm{ng} / \mathrm{mL}$. Houve $27 \%$ de cura no esquema de uma vez por semana, $34 \%$ no grupo tratado duas vezes por semana e $47 \%$ no tratamento diário. Houve fracasso terapêutico (hemoglobina $<10 \mathrm{~g} / \mathrm{dL}$ ) em $41,6,13,2$ e 2,0\% dos casos em cada grupo, respectivamente. Abandono do tratamento por queixa de diarréia ou dor epigástrica só foi observado no tratamento diário.

Conclusão. O tratamento diário continua sendo o mais eficaz. Contudo, o tratamento com sulfato ferroso duas vezes por semana é uma alternativa em caso de dificuldade de adesão ao tratamento diário.

Palavras-chave Anemia ferropriva, anemia ferropriva/terapia, cuidado pré-natal, gravidez, sulfato ferroso.

1 Instituto Materno Infantil de Pernambuco (IMIP). Correspondência e pedidos de separatas devem ser enviados a Ariani Impieri de Souza no seguinte endereço: Instituto Materno Infantil de Pernambuco, Departamento de Pesquisas, Rua dos Coelhos 300, Boa Vista, CEP 50070-550, Recife, PE, Brasil. Telefone: +55-81-2122-4113; telefone/fax:
+55-81-2122-4702; e-mail: saudedamulher@imip. org.br/arianii@terra.com.br/pesquisa@imip.org.br

2 Universidade Federal de Pernambuco (UFPE), Departamento de Nutrição.

3 Universidade de Pernambuco (UPE), Faculdade de Ciências Médicas, Departamento de Medicina Social.
Estima-se que aproximadamente a metade das gestantes no mundo tenha anemia por deficiência de ferro, principalmente nos países em desenvolvimento (1). Embora a deficiência de ferro e a anemia ferropriva na gestação 
sejam citadas como o problema hematológico mais comum do ciclo gestatório, sua prevalência não é conhecida, nem mesmo nos países desenvolvidos, uma vez que a maioria dos estudos investigaram grupos restritos, com acesso a serviços de saúde, não representando a população como um todo (2). No Brasil, a escassez de estudos com amostras representativas (2) não permite a verificação do cumprimento da meta estabelecida na Reunião da Cúpula Mundial em Favor da Infância em 1990, que propôs reduzir em um terço a prevalência de anemia em mulheres na idade reprodutiva (3). Entre as conseqüências da anemia no período da gestação, destacam-se, particularmente, prematuridade e baixo peso ao nascer $(1,4)$. O aumento da mortalidade materna e perinatal também tem sido citado (5), embora não se saiba a partir de que concentração de hemoglobina $(\mathrm{Hb})$ esse efeito ocorreria (6-8).

As medidas de combate à deficiência de ferro e à anemia ferropriva estão bem estabelecidas, consistindo, resumidamente, em modificação dos hábitos alimentares; diagnóstico e tratamento das causas da perda crônica de sangue; controle de infecções e infestações que contribuem para a gênese e o agravamento da anemia; fortificação de alimentos e suplementação medicamentosa com sais de ferro $(1,5)$. O que ainda se procura é a forma mais eficiente de realizar a suplementação com sais de ferro $(9,10)$.

$\mathrm{O}$ questionamento sobre os esquemas habitualmente recomendados de suplementação ocorre em virtude de sua baixa resolubilidade, seja pelo freqüente abandono devido aos efeitos colaterais $(11,12)$, seja pela reduzida absorção do ferro com os esquemas diários e de altas doses habitualmente prescritos.

Os primeiros ensaios clínicos para testar as formas intermitentes de administração de ferro começaram a ser publicados na década de 1990. Esses estudos descreviam experiências em crianças pré-escolares, adolescentes, mulheres em idade fértil e gestantes (13-16), e encontraram respostas semelhantes em termos da elevação da concentração da $\mathrm{Hb}$ tanto com o esquema semanal quanto com o diário. Justificase, assim, o interesse em estabelecer formas mais eficientes (com menor custo, melhor cobertura e maior adesão) de combate a deficiência de ferro e anemia ferropriva, principalmente durante a gestação. Em princípio, o tratamento intermitente pode oferecer respostas satisfatórias para um conjunto de requisitos simultâneos $(1,17)$.

Assim, o objetivo deste estudo foi avaliar se o uso de sulfato ferroso uma ou duas vezes por semana teria a mesma efetividade do uso diário no combate à anemia durante a gestação. Para esta avaliação, foram comparadas as concentrações de $\mathrm{Hb}$, volume corpuscular médio (VCM) e ferritina em grupos submetidos a diferentes esquemas de tratamento.

\section{MATERIAIS E MÉTODOS}

Foi realizado um estudo tipo ensaio clínico, controlado, aleatório e cego do ponto de vista laboratorial, comparando três esquemas de tratamento para anemia em gestantes atendidas no Centro de Atenção à Mulher do Instituto Materno Infantil de Pernambuco (IMIP), na Cidade do Recife, Brasil, no período de maio de 2000 a dezembro de 2001. Em geral, a clientela atendida no IMIP é composta por mulheres de baixa renda e provenientes da própria Cidade do Recife. O índice de desenvolvimento humano (IDH) para o Recife é de 0,644 (nível médio), com $42,9 \%$ dos domicílios ligados à rede geral de esgoto.

A amostra estudada foi composta por gestantes de baixo risco obstétrico atendidas em consulta rotineira de pré-natal, anêmicas ( $\mathrm{Hb}<11 \mathrm{~g} / \mathrm{dL})$ e com 16 a 20 semanas de gestação. Foram excluídas do estudo gestantes com concentração de $\mathrm{Hb}$ abaixo de 8,0 $\mathrm{g} / \mathrm{dL}$ (anemia grave) e as que usaram medicação que continha ferro em sua composição nos 60 dias anteriores à entrada no estudo.

Foram ainda retiradas, no decorrer do estudo, gestantes que relataram uso de qualquer outro medicamento contendo ferro em sua composição, ou que apresentaram algum problema clí- nico que transformasse a gestação em alto risco ou, ainda, mulheres que, nos exames de controle, tivessem registro de concentração de $\mathrm{Hb}$ inferior a 0,5 g/dL em relação à dosagem basal, quando esta fosse abaixo de 10,0 g/dL. Foram excluídas as gestantes que não aderiram a pelo menos $80 \%$ do estabelecido para cada tratamento.

A amostra foi calculada com base na diferença de médias da concentração de $\mathrm{Hb}$ de $0,5 \mathrm{~g} / \mathrm{dL}$ entre os grupos de comparação, com um erro $\alpha$ de 0,05 e um poder de 0,80. Estimou-se assim um valor de 50 mulheres para cada grupo, perfazendo um total de 150 casos a serem observados. A amostra inicial foi de 191 casos. Após as perdas e exclusões ocorridas no decorrer da pesquisa, o estudo foi concluído com 150 gestantes, com 48, 53 e 49 mulheres, respectivamente, nos grupos de tratamento semanal, duas vezes por semana e diário.

Foram utilizadas drágeas de $300 \mathrm{mg}$ de sulfato ferroso heptahidratado $\left(\mathrm{FeSO}_{4} 7 \mathrm{H}_{2} \mathrm{O}\right)$, contendo $60 \mathrm{mg}$ de ferro (Fe) elementar. A duração programada do tratamento foi de 16 semanas ( \pm 1 semana). As gestantes receberam a medicação a cada consulta mensal e foram orientadas a tomar a medicação em casa, com um intervalo de pelo menos 30 minutos em relação à refeição. A cada consulta eram verificadas as drágeas tomadas, por meio de entrevista e contagem dos comprimidos restantes nos frascos. O laboratório de análises desconhecia os esquemas de tratamento, assim como as hipóteses que estavam sendo testadas. Foram analisadas como variáveis: $\mathrm{Hb}$ final, cura de anemia $(\mathrm{Hb}>11 \mathrm{~g} / \mathrm{dL})$, fracasso terapêutico $(\mathrm{Hb}<10 \mathrm{~g} / \mathrm{dL})$, VCM final e ferritina sérica final.

Os questionários foram preenchidos no momento das consultas, diretamente num banco de dados criado no programa Epi Info 6.0 (18).

Foram efetuadas três coletas de sangue (imediatamente antes do início do experimento, 8 semanas após e ao final do experimento) para as dosagens hematológicas. Para a realização do eritrograma, utilizou-se o contador eletrônico de células (Coulter T 890) e, para a análise da ferritina sérica, o método 
Cobas ${ }^{\circledR}$ Core Ferritin EIA do laboratório Roche ${ }^{\circledR}$. Os exames de uroculturas e coproparasitológicos foram solicitados na primeira consulta de pré-natal, conforme a rotina do serviço.

Antes da aplicação dos testes estatísticos adequados para cada situação, verificou-se a compatibilidade dos dados das variáveis hematológicas e não hematológicas com uma distribuição gaussiana (normal). Os testes estatísticos apropriados para cada situação foram definidos com nível de significância de $5 \%$.

Com o consentimento livre e esclarecido das gestantes, o estudo foi aprovado pelo Comitê de Ética do IMIP, que segue como norma referencial a resolução 196/96 do Conselho Nacional de Saúde (19).

\section{RESULTADOS}

Das 393 gestantes recrutadas para participar da pesquisa, $215(54,7 \%)$ eram anêmicas $(\mathrm{Hb}<11 \mathrm{~g} / \mathrm{dL})$. Destas, $88,8 \%(191 / 215)$ aceitaram participar da experiência.

No decorrer do estudo, houve uma perda de seguimento de 21,5\% (41/ 191) por saída espontânea ou exclusão do ensaio. Conforme demonstrado na tabela 1, as perdas se distribuíram igualmente nos três grupos de tratamento $(P=0,551)$. O motivo da perda (abandono ou exclusão da pesquisa) para as 41 gestantes é descrito na tabela 2 . Salienta-se que no grupo tratado uma vez por dia nenhuma gestante teve de ser retirada por redução na concentração da $\mathrm{Hb}$. Por outro lado, este foi o único grupo no qual as gestantes acusaram os efeitos colaterais como motivo do abandono da pesquisa.

A idade das gestantes variou de 13 a 38 anos, com mediana de 22 anos. Quanto à escolaridade, observou-se uma mediana de 9 anos de estudo. A maioria encontrava-se na primeira gestação $(65,3 \%)$. Entre as mulheres que já tinham tido filhos, o intervalo interpartal acima de 2 anos foi observado em $66,0 \%$. Quanto aos problemas clínicos encontrados no decorrer da gestação, sem necessariamente transformar o processo em gestação de alto risco,
TABELA 1. Distribuição das perdas das gestantes com anemia segundo grupo de tratamento, Recife (PE), Brasil, 2000 e 2001

\begin{tabular}{lcccccccr}
\hline & \multicolumn{2}{c}{ Perdas } & & \multicolumn{2}{c}{ Concluíram } & & \multicolumn{2}{c}{ Total } \\
\cline { 2 - 3 } Tratamento (60 mg Fe) & No. & $\%$ & & No. & $\%$ & & No. & $\%$ \\
\hline Uma vez por semana & 16 & 25,0 & & 48 & 75,0 & & 64 & 100,0 \\
Duas vezes por semana & 11 & 17,2 & & 53 & 82,8 & & 64 & 100,0 \\
Uma vez ao dia & 14 & 22,2 & & 49 & 77,8 & & 63 & 100,0 \\
$\quad$ Total & 41 & 21,5 & & 150 & 78,5 & & 191 & 100,0 \\
\hline
\end{tabular}

$\chi^{2}$ (Pearson): $P=0,551$. foram registradas enteroparasitose em 37,3\% das mulheres (ancilostomídeos em $1,3 \%$ ) e infecção do trato urinário em $25,3 \%$. Cerca da metade $(49,3 \%)$ das gestantes estava com o peso adequado, e 30,0\% encontravam-se abaixo do peso para a idade gestacional.

Uma concentração de $\mathrm{Hb}$ entre 10,0 e 10,9 g/dL foi observada em 70,0\% das gestantes. Apenas 6,0\% apresentaram microcitose (VCM $<80 \mathrm{fL}$ ), não aparecendo nenhum caso de macrocitose (VCM > $100 \mathrm{fL}$ ). Ferritina sérica abaixo de $12 \mathrm{ng} / \mathrm{mL}$ foi observada em $15,5 \%$.

Antes de iniciar o tratamento, os grupos eram homogêneos em relação a $\mathrm{Hb}$, VCM e ferritina sérica (tabelas 3 a 5). As variáveis idade, escolaridade, altura, peso, número de gestações, presença de enteroparasitoses e infecção urinária, estado nutricional e idade gestacional também foram homogêneas (tabela 6).

Não houve efeito estatisticamente significativo nas concentrações de $\mathrm{Hb}$ no grupo que utilizou $60 \mathrm{mg}$ de Fe elementar uma vez por semana. Já para os tratamentos que utilizaram o sulfato ferroso duas vezes por semana e uma vez por dia houve elevação estatisticamente significativa na concentração média da $\mathrm{Hb}(0,5$ e 0,9 g/dL, respectivamente) como se descreve na tabela 3 .

A distribuição homogênea das concentrações de $\mathrm{Hb}$ nos três grupos antes do tratamento passou a apresentar resultados diferenciados na avaliação final do experimento, com $27 \%$ de cura no esquema de uma vez por semana, $34 \%$ no grupo tratado duas vezes por semana e $47 \%$ no tratamento diário. Houve fracasso terapêutico $(\mathrm{Hb}$
TABELA 2. Motivo da perda ou exclusão das 41 gestantes com anemia que saíram do estudo, Recife (PE), Brasil, 2000 e 2001

\begin{tabular}{|c|c|c|c|c|}
\hline \multirow[b]{2}{*}{ Motivo } & \multicolumn{3}{|c|}{ Tratamento (60 mg Fe) } & \multirow[b]{2}{*}{ Total } \\
\hline & $\begin{array}{c}\text { Uma vez } \\
\text { por semana }\end{array}$ & $\begin{array}{l}\text { Duas vezes } \\
\text { por semana }\end{array}$ & $\begin{array}{l}\text { Uma vez } \\
\text { ao dia }\end{array}$ & \\
\hline \multicolumn{5}{|l|}{ Abandono } \\
\hline Sem referir motivo & 7 & 5 & 2 & 14 \\
\hline Diarréia/dor epigástrica & 0 & 0 & 7 & 7 \\
\hline \multicolumn{5}{|l|}{ Exclusão } \\
\hline Queda da hemoglobina ${ }^{a}$ & 5 & 3 & 0 & 8 \\
\hline Trabalho de parto prematuro & 2 & 1 & 3 & 6 \\
\hline Uso de polivitamínicos & 1 & 1 & 0 & 2 \\
\hline Gemelaridade & 0 & 1 & 1 & 2 \\
\hline Doença hipertensiva & 1 & 0 & 1 & 2 \\
\hline Total & 16 & 11 & 14 & 41 \\
\hline
\end{tabular}


TABELA 3. Efeito do tratamento na média de concentração de hemoglobina em gestantes com anemia, Recife (PE), Brasil, 2000 a 2001

\begin{tabular}{|c|c|c|c|c|c|c|}
\hline \multirow[b]{2}{*}{ Tratamento $(60 \mathrm{mg} \mathrm{Fe})$} & \multirow[b]{2}{*}{ No. } & \multicolumn{2}{|c|}{ Hemoglobina em g/dL } & \multirow[b]{2}{*}{$\begin{array}{l}\text { Diferença das medias } \\
\text { (depois-antes) }\end{array}$} & \multirow[b]{2}{*}{ IC 95\% } & \multirow[b]{2}{*}{$\begin{array}{c}P \\
\text { (bicaudal) }\end{array}$} \\
\hline & & $\begin{array}{c}\text { Antes } \\
\text { (média } \pm \mathrm{DP})\end{array}$ & $\begin{array}{c}\text { Depois } \\
\text { (média } \pm \mathrm{DP})\end{array}$ & & & \\
\hline Duas vezes por semana & 53 & $10,2 \pm 0,6$ & $10,7 \pm 0,9$ & 0,5 & 0,29 a 0,75 & $<0,001$ \\
\hline Uma vez ao dia & 49 & $10,1 \pm 0,6$ & $11,0 \pm 0,7$ & 0,9 & 0,62 a 1,10 & $<0,001$ \\
\hline ANOVA & & $P=0,518$ & $P=0,001$ & & & \\
\hline
\end{tabular}

a Teste $t$ de Student.

TABELA 4. Efeito do tratamento no volume corpuscular médio em gestantes com anemia, Recife (PE), Brasil, 2000 a 2001

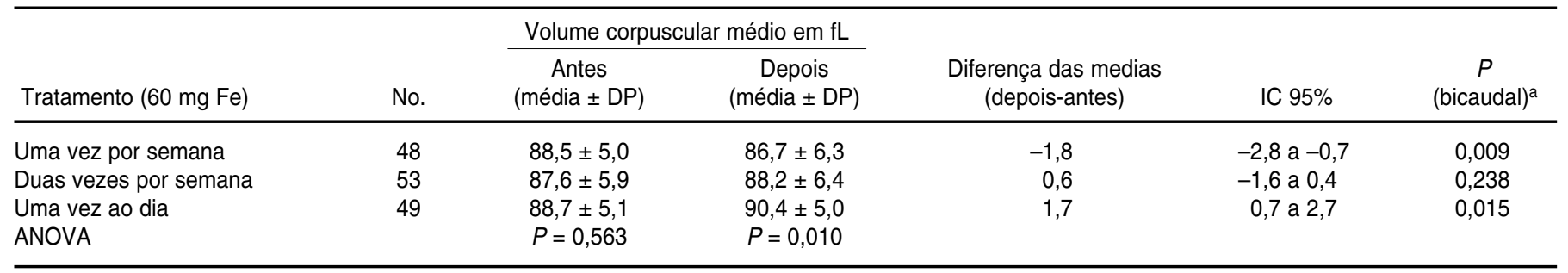

a Teste $t$ de Student.

TABELA 5. Efeito do tratamento sobre a variação das medianas de ferritina em gestantes com anemia, Recife (PE), Brasil, 2000 a 2001

\begin{tabular}{|c|c|c|c|c|c|c|}
\hline \multirow[b]{2}{*}{ Tratamento (60 mg Fe) } & \multirow[b]{2}{*}{ No. } & \multicolumn{2}{|c|}{ Ferritina em ng/mL } & \multirow[b]{2}{*}{$\begin{array}{l}\text { Mediana das diferenças } \\
\text { (antes-depois) }\end{array}$} & \multirow[b]{2}{*}{ IC $95 \%$} & \multirow[b]{2}{*}{$\begin{array}{c}P \\
\text { (bicaudal)a }^{\mathrm{a}}\end{array}$} \\
\hline & & $\begin{array}{c}\text { Antes } \\
\text { (Mediana) }\end{array}$ & $\begin{array}{c}\text { Depois } \\
\text { (Mediana) }\end{array}$ & & & \\
\hline Uma vez por semana & 34 & 30,2 & 8,2 & 25,9 & $-37,1 \mathrm{a}-14,8$ & $<0,001$ \\
\hline Duas vezes por semana & 44 & 37,1 & 12,4 & 28,3 & $-41,2 \mathrm{a}-19,6$ & $<0,001$ \\
\hline Uma vez ao dia & 32 & 52,9 & 15,5 & 33,5 & $-49,4 a-18,6$ & $<0,001$ \\
\hline Kruskal-Wallis & & $P=0,344$ & $P=0,008$ & & & \\
\hline
\end{tabular}

a Teste de Wilcoxon com postos sinalizados.

manteve-se abaixo de $10 \mathrm{~g} / \mathrm{dL}$ ) em $41,6,13,2$ e $2,0 \%$ dos casos em cada grupo, respectivamente (tabela 7).

Em relação ao efeito das intervenções sobre o VCM, observou-se um decréscimo significativo no VCM após o tratamento no grupo que recebeu sulfato ferroso uma vez por semana, ao contrário do grupo diário, que obteve elevação significativa na avaliação final do tratamento. No grupo sob tratamento duas vezes por semana, o aumento do VCM não foi significativo (tabela 4).

Comparando-se o efeito dos esquemas de tratamento sobre as medianas da ferritina sérica, constatou-se que as medianas finais da ferritina foram significativamente menores do que as medianas iniciais nos três esquemas. O teste de Kruskal-Wallis mostrou que a distribuição da ferritina não foi homogênea entre os três grupos no momento final do estudo (tabela 5).

\section{DISCUSSÃO}

A perspectiva de um esquema simplificado de tratamento das anemias constitui por si um objetivo instigante e ambicioso para a assistência pré- natal, principalmente ao se considerar o sulfato ferroso, de custo relativamente baixo e de uso muito difundido, como o fármaco eleito para a concretização dessa proposta. A baixa eficiência dos diferentes regimes terapêuticos utilizados ao longo de décadas tem sido objeto de preocupação das autoridades de saúde. Várias possibilidades têm sido cogitadas para explicar esta ocorrência, desde falta de eficácia dos próprios esquemas utilizados, passando pelos diferentes sais de ferro, até a falta de adesão aos tratamentos, principalmente pela ocorrência de efeitos colaterais atribuídos ao 
TABELA 6. Valores e testes de homogeneidade das variáveis de controle antes do experimento para três grupos de gestantes com anemia, Recife (PE), Brasil, 2000 a 2001

\begin{tabular}{|c|c|c|c|c|}
\hline \multirow[b]{2}{*}{ Variáveis de controle da gestante } & \multicolumn{3}{|c|}{ Tratamento (60 mg Fe) } & \multirow[b]{2}{*}{$P^{a}$} \\
\hline & $\begin{array}{l}\text { Uma vez } \\
\text { por semana } \\
(\text { No. }=48)\end{array}$ & $\begin{array}{c}\text { Duas vezes } \\
\text { por semana } \\
\text { (No. = 53) }\end{array}$ & $\begin{array}{c}\text { Uma vez } \\
\text { ao dia } \\
\text { (No. = 49) }\end{array}$ & \\
\hline Mediana (DIQ) $)^{b}$ de idade em anos & $21(8,5)$ & $22(7,0)$ & $22(8,0)$ & 0,306 \\
\hline Mediana (DIQ) ${ }^{\mathrm{b}}$ de escolaridade em anos & $9(5,0)$ & $9(4,0)$ & $9(4,0)$ & 0,642 \\
\hline Média (DP) de altura em $\mathrm{cm}$ & $156,8 \pm 5,7$ & $156,9 \pm 5,5$ & $156,0 \pm 6,3$ & 0,686 \\
\hline Média (DP) de peso em kg & $59,3 \pm 11,5$ & $59,7 \pm 10,9$ & $57,7 \pm 10,2$ & 0,624 \\
\hline Mediana (DIQ) $)^{b}$ do número de gestações & $1(1,0)$ & $1(1,0)$ & $1(1,0)$ & 0,453 \\
\hline Enteroparasitoses (\%) & 44,7 & 31,3 & 41,7 & 0,370 \\
\hline Infecção urinária (\%) & 16,7 & 26,9 & 32,7 & 0,188 \\
\hline \multicolumn{5}{|l|}{ Estado nutricional (\%) } \\
\hline Baixo peso & 27,1 & 30,2 & 32,7 & 0,942 \\
\hline Peso adequado & 54,2 & 47,2 & 46,9 & \\
\hline Sobrepeso/obesidade & 18,8 & 22,7 & 20,4 & \\
\hline \multicolumn{5}{|l|}{ Média da idade gestacional em semanas } \\
\hline No diagnóstico & 17,3 & 17,5 & 17,3 & 0,586 \\
\hline No início do tratamento & 21,1 & 21,1 & 21,0 & 0,891 \\
\hline
\end{tabular}

${ }^{a}$ ANOVA para comparar médias, Kruskal-Wallis para comparar medianas e $\chi^{2}$ para comparar proporções.

b Distância interquartil = Q3-Q1

ferro, em particular dor abdominal, diarréia e constipação $(3,5)$.

Nos últimos 10 anos, tem sido sugerida a possibilidade de tratamentos intermitentes com ferro como alternativa para melhorar a adesão e, conseqüentemente, a própria resposta ao tratamento. Estudos em crianças e mulheres não grávidas, utilizando esses esquemas, têm mostrado resultados promissores $(13,14,16)$.
Neste estudo, os resultados obtidos com a dose única semanal de sulfato ferroso indicam uma resposta satisfatória em apenas $27,1 \%$ dos casos, com a restauração das concentrações de $\mathrm{Hb}$ para o limite de normalidade $(\mathrm{Hb}>$ $11,0 \mathrm{~g} / \mathrm{dL}$ ). Esse percentual de cura está aquém da meta desejável, que seria a redução de pelo menos um terço na prevalência de anemia em mulheres (3). Ademais, no final do tra-

TABELA 7. Distribuição da concentração de hemoglobina segundo esquema de tratamento de gestantes com anemia, Recife (PE), 2000 a 2001

\begin{tabular}{|c|c|c|c|c|c|c|c|c|}
\hline \multirow[b]{3}{*}{$\mathrm{Hb}(\mathrm{g} / \mathrm{dL})$} & \multicolumn{6}{|c|}{ Tratamento com $60 \mathrm{mg} \mathrm{Fe}$} & & \\
\hline & \multicolumn{2}{|c|}{$\begin{array}{c}\text { Uma vez } \\
\text { por semana }\end{array}$} & \multicolumn{2}{|c|}{$\begin{array}{l}\text { Duas vezes } \\
\text { por semana }\end{array}$} & \multicolumn{2}{|c|}{$\begin{array}{l}\text { Uma vez } \\
\text { ao dia }\end{array}$} & \multicolumn{2}{|c|}{ Total } \\
\hline & No. & $\%$ & No. & $\%$ & No. & $\%$ & No. & $\%$ \\
\hline \multicolumn{9}{|c|}{ Antes do tratamento ${ }^{a}$} \\
\hline 8,0 a 9,9 & 15 & 31,2 & 14 & 26,4 & 16 & 32,7 & 45 & 30,0 \\
\hline 10,0 a 10,9 & 33 & 68,8 & 39 & 73,6 & 33 & 67,3 & 105 & 70,0 \\
\hline Total & 48 & 100,0 & 53 & 100,0 & 49 & 100,0 & 150 & 100,0 \\
\hline \multicolumn{9}{|c|}{ Após o tratamento b } \\
\hline 8,0 a 9,9 & 20 & 41,6 & 07 & 13,2 & 01 & 2,0 & 28 & 18,7 \\
\hline 10,0 a 10,9 & 15 & 31,3 & 28 & 52,8 & 25 & 51,0 & 68 & 45,3 \\
\hline$\geq 11,0$ & 13 & 27,1 & 18 & 34,0 & 23 & 47,0 & 54 & 36,0 \\
\hline Total & 48 & 100,0 & 53 & 100,0 & 49 & 100,0 & 150 & 100,0 \\
\hline
\end{tabular}

\footnotetext{
a Teste de Jonckheere-Terpstra: $P=0,876$.
}

b Teste de Jonckheere-Terpstra: $P<0,001$.

tamento, 41,6\% das gestantes que receberam ferro uma vez por semana apresentaram concentrações de $\mathrm{Hb}$ entre 8,0 g/dL e 9,9 g/dL, caracterizando uma elevada prevalência de anemia $(8,20,21)$. Além disso, este grupo apresentou uma redução na média do VCM de 88,5 \pm 5,0 para 86,7 $\pm 6,3 \mathrm{fL}$. Tal diferença foi estatisticamente significativa, embora se mantendo ainda dentro da faixa considerada normal (VCM entre 80,0 e 100 fL). Outra constatação de caráter adverso refere-se ao fato de que, na avaliação final, a mediana de ferritina sérica neste grupo foi de $8,2 \mathrm{ng} / \mathrm{mL}$, bem abaixo, portanto, do limite inferior aceito como normal (12,0 ng/mL) (4). Como achado positivo, ressalva-se que nenhuma perda por abandono do tratamento neste grupo foi atribuída pelas gestantes aos efeitos supostamente indesejáveis da medicação.

Já os resultados terapêuticos obtidos com o tratamento que utilizou sulfato ferroso duas vezes por semana foram bem mais promissores. Assim, a concentração média de $\mathrm{Hb}$ ao final do tratamento alcançou a elevação mínima preestabelecida de 0,5 g/dL, sendo estatisticamente significativa. $\mathrm{O}$ percentual de cura alcançou o índice de 
$34,0 \%$, atingindo, assim, a recomendação do UNICEF (3). Apenas 13,2\% permaneceram na categoria considerada como fracasso terapêutico; a média do VCM se manteve e a mediana da ferritina também se manteve acima do ponto de corte de $12 \mathrm{ng} / \mathrm{mL}$. Isto sugere haver elevada mobilização do ferro estocado durante a gestação, mesmo nas gestantes submetidas à suplementação (1).

No esquema de tratamento proposto como controle (sulfato ferroso diário), $47,0 \%$ das gestantes foram consideradas curadas. Em termos comparativos, os resultados obtidos com este esquema não diferiram estatisticamente dos valores obtidos com o esquema que utilizou sulfato ferroso duas vezes por semana. No entanto, ao final do tratamento diário, apenas 2,0\% dos casos foram classificados como fracassos terapêuticos, enquanto a média do VCM se elevou significativamente. Por fim, a mediana final de ferritina sérica se situou acima do nível crítico indicativo de depleção substancial nas reservas orgânicas de ferro (12,0 ng/ $\mathrm{mL}$ ). Em contraposição aos resultados terapêuticos positivos neste grupo de tratamento, $50 \%$ perdas ocorridas a partir da amostra inicial foram atribuídas aos efeitos secundários da medicação, mais explicitamente a dor epigástrica e diarréia.

Considerando os aspectos comparativos intergrupos, no que se refere ao objetivo dominante (correção ou cura da anemia), os resultados expressos em percentuais de cura evidenciam a melhor resposta terapêutica com o uso mais intenso (diário) da medicação. Essa observação é reforçada pelo per- centual de fracasso terapêutico, que diminui com o aumento na freqüência de ingestão do sulfato ferroso.

Um dos estudos pioneiros no sentido de encontrar um tratamento alternativo simplificado para a anemia em gestantes foi o de Ridwan et al. (15), que utilizou sulfato ferroso na forma diária (60 mg de $\mathrm{Fe}$ elementar) e na forma semanal (120 mg de Fe elementar) durante uma média de 14 semanas. Os autores observaram uma elevação significativa semelhante na concentração de $\mathrm{Hb}$ nos dois esquemas, sendo que, quando apenas as mulheres anêmicas foram analisadas, nenhum dos dois grupos alcançou a concentração média de $\mathrm{Hb}$ indicativa de cura. Nessas condições, ambos os esquemas de tratamento foram considerados por Hallberg como ineficientes (17).

Mais recentemente, um outro ensaio (21), com desenho semelhante ao do presente estudo, comparou a utilização de $60 \mathrm{mg}$ de ferro diário com 60 mg de ferro duas vezes por semana. Como resultado, no tratamento diário a concentração média da $\mathrm{Hb}$ elevou-se de 9,3 para $11,4 \mathrm{~g} / \mathrm{dL}$; com ingestão de ferro duas vezes por semana, a concentração média da $\mathrm{Hb}$ subiu de 9,6 para $10,7 \mathrm{~g} / \mathrm{dL}$. No entanto, ao final do experimento, a diferença entre os dois tratamentos foi estatisticamente significativa em favor do esquema diário. Salienta-se, ademais, que o tratamento diário também elevou a ferritina sérica na avaliação final, o que não ocorreu no presente ensaio.

Na Malásia, Young et al. (22) também compararam dois esquemas de tratamento para anemia em gestantes (60 mg de Fe elementar diário versus
$120 \mathrm{mg}$ de Fe elementar uma vez por semana), não encontrando diferenças entre os esquemas. Vale observar que, naquele estudo, nenhum dos esquemas de tratamento elevou a concentração média de $\mathrm{Hb}$ para o nível da cura.

A propósito dos limites de cura, discute-se se seria necessário elevar a concentração da $\mathrm{Hb}$ até o limite atualmente recomendado de $11,0 \mathrm{~g} / \mathrm{dL}$, considerando fracasso terapêutico uma concentração < 10,0 g/dL. Sabese que uma concentração de $\mathrm{Hb}$ entre 10,0 e 10,9 g/dL não está associada a resultados adversos, seja para a mãe, seja para o bebê $(6,8,20,23)$. Assim, esse patamar poderia ser considerado como limítrofe de cura.

Em termos conclusivos, três apreciações devem ser ressaltadas: 1) não é recomendável tratar a anemia em gestantes com aplicações semanais de ferro; 2) o esquema diário seria o padrão-ouro se não fosse pela verificação de que grande parte das gestantes desistiram do tratamento pela ocorrência de efeitos colaterais indesejáveis; 3) o esquema que utilizou ferro duas vezes por semana obteve um percentual de cura aceitável (34\%), mas apresentou um percentual muito alto de fracasso terapêutico $(13,2 \%)$, tendo a seu favor a ressalva de que nenhuma gestante desse grupo interrompeu o tratamento pela ocorrência de efeitos colaterais inaceitáveis. Diante dessa situação, poderia ser mantida a recomendação do esquema de uso diário de sulfato ferroso, com a alternativa de se continuar o tratamento com o esquema de sulfato ferroso duas vezes por semana nos casos em que ocorra intolerância ao plano terapêutico de rotina.

\section{REFERÊNCIAS}

1. World Health Organization. Iron deficiency anaemia: assessment, prevention, and control: a guide for programme managers. Geneva: WHO; 2001.

2. Santos LMP, org. Bibliografia sobre deficiência de micronutrientes no Brasil: 1990-2000. Brasília: Organização Pan-Americana da Saúde; 2002.

3. Fundo das Nações Unidas para a Infância. Estratégia para melhorar a nutrição de crianças e mulheres nos países em desenvolvimento. Nova Iorque: UNICEF; 1990.

4. Scholl TO, Hediger ML, Fischer RL, Shearer JW. Anemia versus iron deficiency: increased risk of preterm delivery in a prospective study. Am J Clin Nutr. 1992;55(5):985-8.

5. United Nations Children's Fund. Preventing iron deficiency in women and children: technical consensus on key issues. Nova Iorque: UNICEF; 1998.
6. Mahomed K, Hytten F. Iron and folate supplementation in pregnancy. Em: Chalmers I, Enkin M, Keirse MJNC. Effective care in pregnancy and childbirth. Oxford: Oxford University Press; 1993.

7. Scholl TO, Reilly T. Anemia, iron and pregnancy outcome. J Nutr. 2000;130:443S-447S. Disponível em: http://www.nutrition.org/ cgi/content/full/130/2/443S. Acessado em abril de 2004. 
8. Scanlon KS, Yip R, Schieve LA, Cogswell ME. High and low hemoglobin levels during pregnancy: differential risks for preterm birth and small for gestational age. Obstet Gynecol. 2000;96(5):741-8.

9. Mahomed K. Iron supplementation in pregnancy (Cochrane Review). Em: The Cochrane Library. Issue 2. Oxford: Update Software; 2003.

10. Hallberg L, Reply TO, Shultink W, Gross R. Use of daily compared with weekly iron supplementation: apples and pears [letter]. Am J Clin Nutr. 1999;69(4):739-42.

11. Sjöstedt JE, Manner P, Nummi S, Ekenved G. Oral iron prophylaxis during pregnancy a comparative study on different dosage regimens. Acta Obstet Gynecol Scand. 1977; 60(suppl):3-9.

12. Batista Filho M, Ferreira LOC. Prevenção e tratamento da anemia nutricional ferropriva: novos enfoques e perspectivas. Cad Saude Publica. 1996;12(3):411-5.

13. Schultink W, Gross R, Gliwitzki M, Karyadi D, Matulessi P. Effect of daily vs. weekly iron supplementation in Indonesian preschool children with low iron status. Am J Clin Nutr. 1995;61(1):111-5.

14. Angeles-Agdeppa I, Schultink W, Sastroamidjojo S, Gross R, Karyadi D. Weekly mi- cronutrient supplementation to build iron stores in female Indonesian adolescents. Am J Clin Nutr. 1997;66(1):177-83.

15. Ridwan E, Schultink W, Dillon D, Gross R. Effects of weekly iron supplementation on pregnant Indonesian women are similar to those of daily supplementation. Am J Clin Nutr. 1996;63(6):884-90.

16. Lopes MCS, Ferreira LOC, Batista Filho M. Uso diário e semanal de sulfato ferroso no tratamento de anemia em mulheres no período reprodutivo. Cad Saude Publica. 1999;15(4): 799-808.

17. Hallberg L. Combating iron deficiency: daily administration of iron is far superior to weekly administration. Am J Clin Nutr. 1998; 68(2):213-7.

18. Dean AG, Dean JA, Coulombier D, Brendel KA, Smith DC, Burton AH, et al. Epi Info, Version 6.0: a word processing database, and statistics program for epidemiology on microcomputers. Centers of Disease Control and Prevention, Atlanta, Georgia, USA; 1994.

19. Brasil, Ministério da Saúde, Conselho Nacional de Saúde. Normas de pesquisa envolvendo seres humanos. Resolução CNS 196/ 96. Bioética 1996;4(2 Supl):15-25. Disponível em: http://www.portalmedico.org.br/revista/ ind2sv4.htm. Acessado em abril de 2004.
20. Murphy JF, O'Riordan J, Newcombe RG Coles EC, Pearson JF. Relation of haemoglobin levels in first and second trimesters to outcome of pregnancy. Lancet. 1986;1(8488): 992-5.

21. Mumtaz Z, Shahab S, Butt N, Rab AR, DeMuynck A. Daily iron supplementation is more effective than twice weekly iron supplementation in pregnant women in Pakistan in a randomized double-blind clinical trial. J Nutr. 2000;130(11):2697-702.

22. Young MW, Lupafya E, Kapenda E, Bobrow EA. The effectiveness of weekly iron supplementation in pregnant women of rural Northern Malawi. Trop Doct. 2000;30(2):84-8.

23. Beaton GH. Iron needs during pregnancy: do we need to rethink our targets? Am J Clin Nutr. 2000;72(suppl):265S-71S. Disponível em: http://www.micronutrient.org/idpas/ pdf/661IronNeeds.pdf. Acessado em abril de 2004.

Manuscrito recebido em 21 de julho de 2004. Aceito em versão revisada em 28 de janeiro de 2004

ABSTRACT Objective. To evaluate the effectiveness of three regimens employing ferrous sulfate to treat pregnant women with anemia.

Methods. The study was carried out at the Women's Health Center of the PernamThe effectiveness of three regimens using ferrous sulfate to treat anemia buco Institute of Maternal and Child Health in the city of Recife, Pernambuco, Brazil, from May 2000 to December 2001. A randomized clinical trial with blinded laboratory analysis was conducted. Iron $(60 \mathrm{mg})$ was administered as $300-\mathrm{mg}$ ferrous sulfate tablets. The women were allocated to three treatment groups, according to the frequency of ingesting the tablets: once a week ( 48 women), twice a week (53 women), and once a day (49 women). The groups were compared for the values for hemoglobin $(\mathrm{Hb})$ concentration, mean corpuscular volume, and ferritin before and after the treatment.

Results. Before the intervention, the groups were homogeneous. They had the following mean ( \pm standard deviation) concentrations of hemoglobin: $10.2 \pm 0.5 \mathrm{~g} / \mathrm{dL}$ for the group receiving iron once a week, $10.2 \pm 0.6 \mathrm{~g} / \mathrm{dL}$ for the group receiving iron twice a week, and $10.1 \pm 0.6 \mathrm{~g} / \mathrm{dL}$ for the group receiving iron once a day. The means of corpuscular volume were, respectively: $88.5 \pm 5.0 \mathrm{fL}, 87.6 \pm 5.9 \mathrm{fL}$, and $88.7 \pm 5.1 \mathrm{fL}$. The respective medians for ferritin were $30.2 \mathrm{ng} / \mathrm{mL}, 37.1 \mathrm{ng} / \mathrm{mL}$, and $52.9 \mathrm{ng} / \mathrm{mL}$. The cure rate $(\mathrm{Hb}>11 \mathrm{~g} / \mathrm{dL})$ was $27 \%$ in the patients treated once a week, $34 \%$ in those treated twice a week, and $47 \%$ in the women treated daily. Treatment failure (hemoglobin $<10 \mathrm{~g} / \mathrm{dL}$ ) was seen in $41.6 \%, 13.2 \%$, and $2.0 \%$ of the patients in the respective groups. Interruption of treatment due to diarrhea or epigastric pain occurred only among the patients treated daily.

Conclusion. The regimen with iron administered daily is still the best option for treating anemia. However, treatment with ferrous sulfate administered twice a week is an alternative for patients who are unable to adhere to daily treatment. 